\title{
First results from the AugerPrime Radio Detector
}

Tomáš Fodran ${ }^{a, *}$ on behalf of the Pierre Auger ${ }^{b}$ Collaboration

(a complete list of authors can be found at the end of the proceedings)

${ }^{a}$ Department of Astrophysics/IMAPP, Radboud University, P.O. Box 9010, NL-6500 GL Nijmegen, The Netherlands

${ }^{b}$ Observatorio Pierre Auger, Av. San Martín Norte 304, 5613 Malargüe, Argentina

E-mail: spokespersons@auger.org

The Pierre Auger Observatory investigates the properties of the highest-energy cosmic rays with unprecedented precision. The aim of the AugerPrime upgrade is to improve the sensitivity to the primary particle type. The improved mass sensitivity is the key to exploring the origin of the highest-energy particles in the Universe. The purpose of the Radio Detector (as part of AugerPrime) is to extend the sensitivity of the mass measurements to zenith angles in the range from $65^{\circ}$ to $85^{\circ}$. A radio antenna, sensitive in two polarization directions and covering a bandwidth from 30 to $80 \mathrm{MHz}$, will be added to each of the 1661 surface detector stations over the full $3000 \mathrm{~km}^{2}$ area, forming the world's largest radio array for the detection of cosmic particles. Since November 2019, an engineering array comprised of ten stations has been installed in the field. The radio antennas are calibrated using the Galactic (diffuse) emission. The sidereal modulation of this signal is monitored continuously and is used to obtain an end-to-end calibration from the receiving antenna to the ADC in the read-out electronics. The calibration method and first results will be presented. The engineering array is also fully integrated in the data acquisition of the Observatory and records air showers regularly. The first air showers detected simultaneously with the water-Cherenkov detectors and the Radio Detectors will be presented. Simulations of the detected showers, based on the reconstructed quantities, have been conducted with CORSIKA/CoREAS. A comparison of the measured radio signals with those predicted by simulations exhibits satisfying agreement.

$37^{\text {th }}$ International Cosmic Ray Conference (ICRC 2021)

July 12th - 23rd, 2021

Online-Berlin, Germany

\footnotetext{
*Presenter
} 


\section{Introduction}

A large upgrade of the Pierre Auger Observatory, named AugerPrime, is currently in progress [1]. After the upgrade, the surface detector stations (SD) will consist of a surface scintillator detector (SSD) [2], a water-Cherenkov detector (WCD) with three original photomultiplier tubes (PMTs) and one new small PMT, and a radio antenna [3, 4]. The installed radio antennas will serve as the Radio Detector (RD). The RD antenna is comprised of two orthogonally oriented short aperiodic loaded loop antennas (SALLA) of $1.2 \mathrm{~m}$ diameter [5]. The RD is triggered by the signal from WCDs. The signals received by the antenna are read-out at the top, where they are also amplified in a low noise amplifier (LNA). Proper matching between the antenna structure and the LNA is realized with a 3:1 transmission line transformer. At the bottom of the antenna is a resistance of $450 \Omega$ to reduce the influence of the reflected radiation from the structures below the antenna. From the LNA, the signals are transmitted through a shielded coaxial cable to a digitizer with a sampling frequency of $250 \mathrm{MHz}$ and bit depth of 12 bits. The stored ADC values from the digitizer are passed through the read-out electronics system at each station to the central data acquisition of the Observatory. As part of AugerPrime, the role of the RD is to detect the radio emission from air showers in the frequency range from 30 to $80 \mathrm{MHz}$, arriving from zenith angles in the range from $65^{\circ}$ to $85^{\circ}$. The RD, in combination with the WCD, will be used to measure the ratio of the electromagnetic energy and the number of muons for horizontal air showers, increasing the aperture of the Observatory for mass-sensitive investigations [6]. The expected performance of the RD can be found in reference [7]. The RD engineering array currently comprised of ten mechanical stations was installed in November 2019. Seven of these stations are equipped with read-out electronics, from which four are used for air-shower measurements.

In the following, we present an end-to-end pilot calibration of the RD using the Galactic radio emission. The motivation is to evaluate the functionality of the RD concept and build up the theoretical and technical framework for a later full-field calibration. Further, we present the first air showers measured with the engineering array and compare them with the simulations based on the reconstructed quantities.

\section{Measured dataset}

For calibration, we use five months of data from one engineering array station located in the regular $1500 \mathrm{~m}$ grid. Its approximate trigger rate is around one event per two hours. The considered dataset for calibration consists of 2775 traces. In addition to the signal from the Galaxy, the measured signal may also contain a cosmic-ray (CR) signal, periodical and transient environmental noise, and internal electronic (thermal) noise. For the calibration, traces containing CR signals or transient radio-frequency interference (RFI) have to be removed from the dataset. To find the cut-out thresholds that would safely remove only traces not suitable for the calibration, we use distributions of basic trace properties (Fig. 1). We set the cut-out thresholds where the fitted distribution's cumulative distribution function (CDF) reaches a chosen value (dashed lines in Fig. 1). In total, we removed $1.1 \%$ of traces. Once the data have been cleaned in this way, they are integrated over $1 \mathrm{MHz}$-wide bins and binned to 1-hour local sidereal time (LST) bins. The power in a frequency interval $[f, f+\delta]$ is calculated with the following expression: 

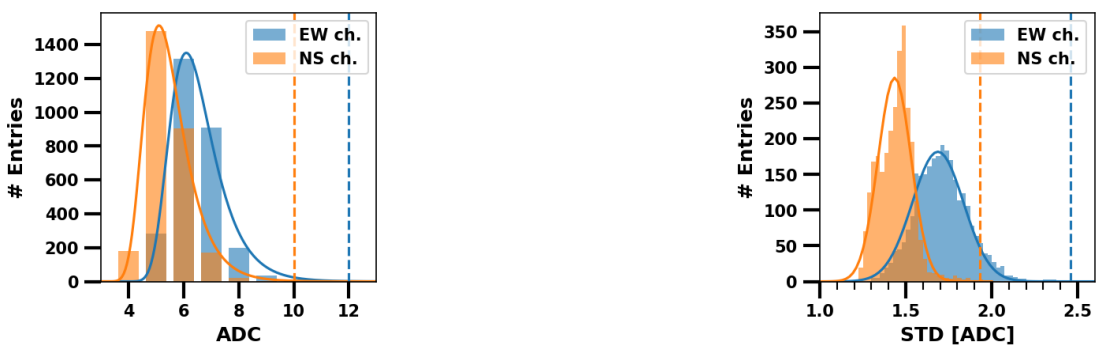

(a) Distribution of the maximal amplitudes of the traces. (b) Distribution of standard deviations of traces samples.

Figure 1: Distribution of basic trace properties in the east-west (EW) and north-south (NS) channels. Fig. (a) is fitted with a Gumbel distribution and Fig. (b) with a Gaussian distribution. The dashed line shows where the $1-\mathrm{CDF}$ of the fitted distribution reaches values of $1^{-3}$ (a) and $1^{-7}$ (b), respectively.

$$
P_{f}=2 \frac{1}{T} \sum_{k=f}^{f+\delta} \frac{|X(k)|^{2}}{R(k)} \Delta f
$$

where $T$ is the time length of the trace, $\Delta f$ is the frequency spectrum resolution, $R(k)$ is the antenna impedance, and $X(k)$ is the single-sided discrete Fourier transform of the signal, normalized by the sampling frequency. The factor 2 in the Eq. (1) is due to the use of the single-sided spectra.

The power dataset, cleaned from the transient RFI and the cosmic-ray signals, includes contributions only from the Galactic signal, the internal electronic noise, and the periodical environmental noise. The internal electronic noise is Johnson-Nyquist noise, from the signal chain components. This is a white noise, independent of frequency and scales linearly with the temperature. However, by propagating through the signal chain, the internal noise inherits frequency dependence from the gain of the signal chain components. When averaging over a whole year, daily temperature variations cancel out in the LST domain, and the internal electronic noise contributes as a LST constant offset to the Galactic signal. If the amplitude of the daily temperature variation of the internal electronic noise is small enough, its LST average can be sufficiently flat even sooner.

The calculated power in the EW channel for all bins as a function of frequency and LST, excluding bands with narrow-band RFI, is shown in Fig. 2(a). The mean standard error of the calculated power bins in both channels is $\sim 4 \%$ ( $2 \%$ in voltage). The semi-broad structures spawning through several frequency bands on an otherwise smooth baseline that can be seen in Fig. 2(a) are caused by the periodical environmental noise. To clean the baseline, we use the algorithm described in [8]. The resulting dataset is illustrated in Fig. 2(b).

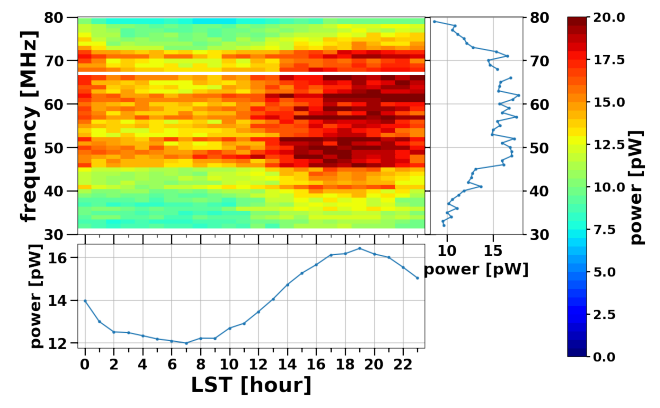

(a) EW channel dataset.

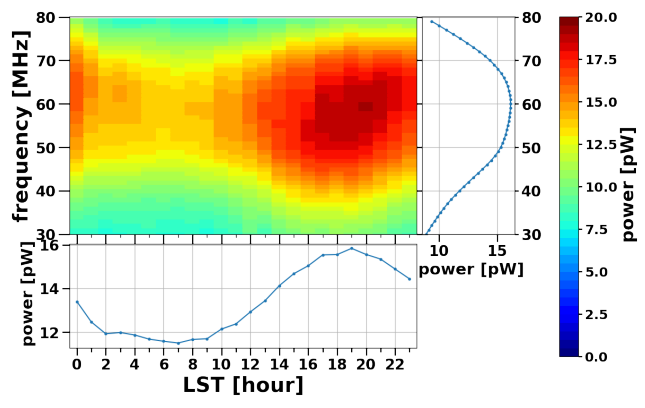

(b) Baseline from the EW channel dataset.

Figure 2: Measured dataset. Fig.(a) Dataset after signal cleaning (see text). Narrow band RFIs are excluded. Fig. (b) Dataset (a) after cleaning from the periodical environmental noise. 


\section{Simulated Galactic signal}

The expected power delivered to the antenna in an interval $[f, f+\delta]$ is calculated as

$$
P_{\text {sky }}(\tau, f)=\frac{2 k_{\mathrm{B}}}{c^{2}} \int_{f}^{f+\delta} f^{\prime 2} \frac{Z_{0}}{R_{r}\left(f^{\prime}\right)} \int_{\Omega} T_{\text {sky }}\left(\theta, \phi, \tau, f^{\prime}\right)\left\langle\left|H\left(f^{\prime}, \theta, \phi\right)\right|^{2}\right\rangle d \Omega d f^{\prime}
$$

where $k_{\mathrm{B}}$ is the Boltzmann constant, $c$ is the speed of light, $Z_{0}$ is the impedance of free space, $R\left(f^{\prime}\right)$ is the antenna model impedance, $T_{\text {sky }}\left(\theta, \phi, f^{\prime}\right)$ is the sky temperature at local coordinates, $f^{\prime}$ is the frequency and $\tau$ is the local sidereal time. The term $\left\langle\left|H\left(f^{\prime}, \theta, \phi\right)\right|^{2}\right\rangle$ is the average antenna response to unpolarized waves [9]. From Eq. (2), we see that the expected power depends on the simulated antenna model and sky temperature.

Sky temperature maps are available as tabulated data: LFmap [10] and PyGDSM [11]. LFmap provides a map for which the user can set the frequency of the spectral bending and HII absorption regions. We use the default settings. An example of a simulated power dataset propagated to the end of the signal chain using LFmap is shown in Fig. 3(a). PyGDSM provides sky maps built on four different references: LFSS [12], GSM2008 [13], GSM2016 [14] and Haslam [15, 16] with settable spectral index. For the Haslam map we set the spectral index to -2.52 [17]. The sky temperature map differences are illustrated in Fig. 4 relative to LFmap. The differences between the different maps are directionally dependent and vary up to $\pm 50 \%$, even more for point sources.

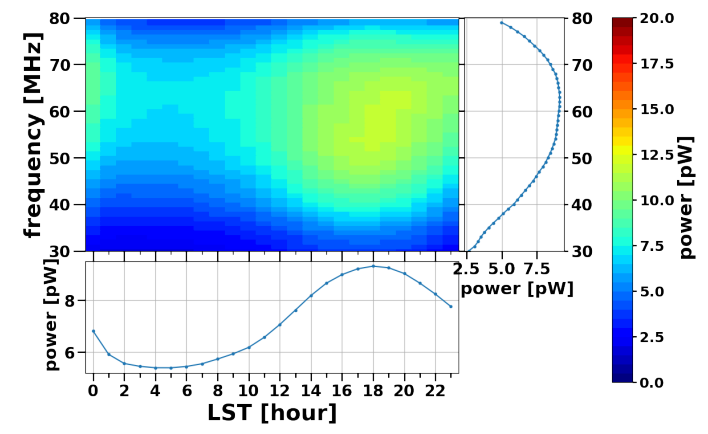

(a) Simulated power in EW Channel.

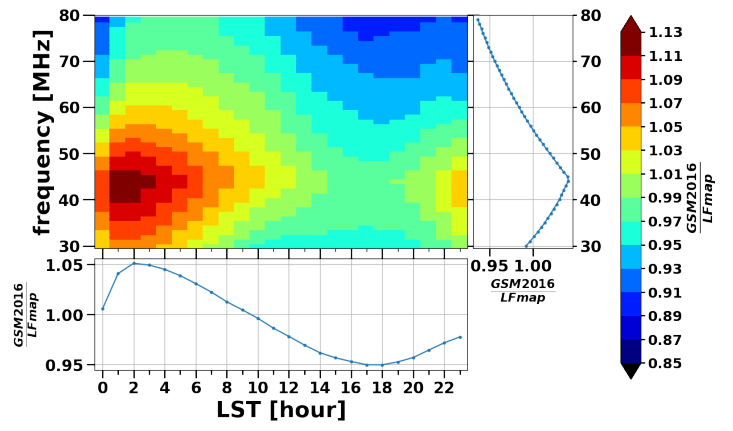

(b) $\frac{\text { GSM2016 }}{\text { LFmap }}$

Figure 3: Left: Simulated power in the EW Channel calculated using the LFmap. Right: Ratio of the simulated power in the EW channel calculated with GSM2016 and LFmap.

The coordinate transformation from the equatorial (or Galactic) to the local coordinate system introduces a time dependence in the expected delivered power. The effect of the differences between the sky temperature maps on the expected power for the case of GSM2016 and LFmap is illustrated in Fig.3(b). Upon the integration over the whole sky, the differences between the sky maps are diminished and influence the total expected power much less (as can be noticed comparing Fig. 4(c) and 3(b)). The LST dependence of the differences between the sky maps seen in Fig. 3(b) is because at different LST, different parts of the sky maps are at zenith where the antenna has the highest gain. For example, the differences in the hot parts (e.g., Galactic center) of the sky map will prevail in the calculated power the most at 18:00 LST (Galactic center in the zenith). Conversely, differences in the cold parts of the maps prevail mostly at early LST hours (e.g., 02:00 LST). The frequency dependence of the differences in Fig. 3(b) is due to the different values of the spectral indices used by the individual sky maps. 
The second factor that affects the expected power is the antenna response. The antenna model is simulated with $4 \mathrm{NEC} 2$. We use simulations of various modifications of our default antenna model to evaluate the effect of the antenna response on the calibration constant.

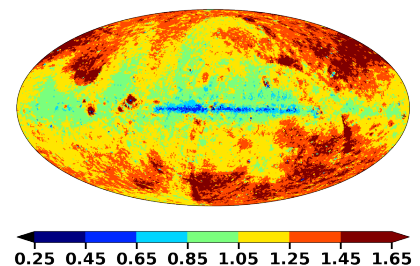

(a) $\frac{\text { LFSS }}{\text { LFmap }}$

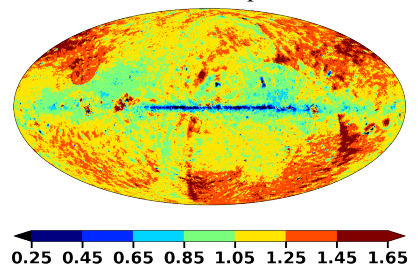

(c) $\frac{\text { GSM2016 }}{\text { LFmap }}$
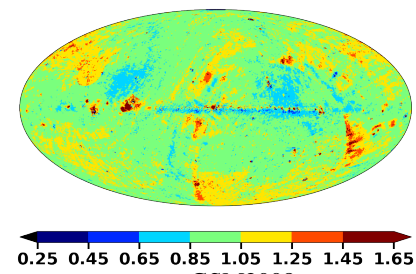

(b) $\frac{\text { GSM2008 }}{\text { LFmap }}$

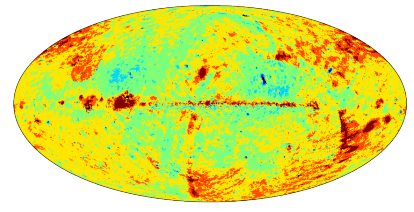

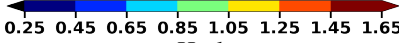

(d) $\frac{\text { Haslam }}{\text { LFmap }}$

Figure 4: Comparison of the sky temperature maps in galactic coordinates at $45 \mathrm{MHz}$. Intensities relative to LFmap are shown.

\section{Calibration methods}

The calibration is necessary to correct for uncertainties in the system response. The simplest way to perform the calibration is to fit the whole measured and simulated datasets with a linear regression [18]. The fitted slope gives the calibration constant and the intersection the amount of background noise. Such an approach implies that the noise at the end of the signal chain is frequency independent, which is not always true. Thermal noise entering the signal chain at different levels inherits frequency dependence from the signal chain components.

The calibration model used by LOFAR takes this feature into account and uses the noise contributions entering at different levels of the signal chain as free parameters while determining the scaling (calibration) constant by minimizing differences between measured and simulated datasets [9]. This approach is more realistic. However, it does not take into account the existence of the environmental periodical noise. To accommodate this feature, for the LOFAR method, we use the dataset with the cleaned baseline described in section 2. The baseline represents the upper bound of the possible thermal noise. We use the LOFAR method in two different approaches. In the first one, we assume that the internal noise constants are identical for each channel, while in the second one, the noise constants are separate for each channel.

Lastly, we introduce a model which fits each band separately with a linear regression. The intersection from each fit then represents a sum of the thermal and external noise directly. Bands containing narrow-band RFI (e.g., $67 \mathrm{MHz}$ in Fig. 2(a)) are excluded. The calibration constant is then given as the average of the fitted slopes. We also use a modified version of this model with an implied condition of the same slope for each band. In this way, one value of the slope is obtained from the fits, but the number of obtained intersections is still equal to the number of bands. 


\section{Calibration results}

We simulate the expected power by using five different sky temperature map models ${ }^{1}$ in combination with 17 different antenna models ${ }^{2}$. In all antenna models, the design of the antenna loop is unchanged. We perform the calibration with four methods discussed above. In total, we get 340 calibration constants, separately for the EW and NS channels. To account for the underlying uncertainty of individual sky maps (10\%[10], 25\%[12], 10\%[13], 15\%[14], 10\%[16]), we smear each individual calibration constant by 1000 random normal numbers with standard deviations according to the quoted sky map uncertainty. The overall distribution of the resulting voltage calibration constants is shown in Fig. 5.

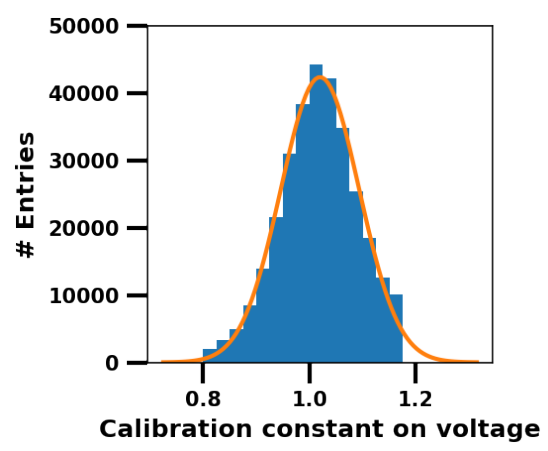

(a) EW Channel.

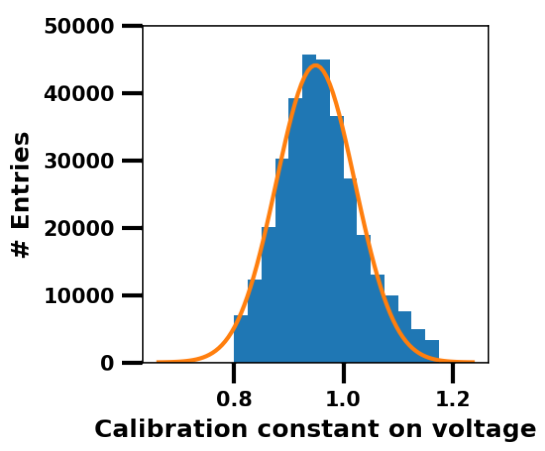

(b) NS Channel.

Figure 5: Distribution of the calibration constants obtained combining different sky temperature maps, antenna models, and calibration methods. The means and standard deviations can be found in Table 1.

The final value of the calibration constant is given as the mean value of the distribution with the standard deviation as the uncertainty, which then includes uncertainties caused by the choice of the sky map, antenna model, calibration model, and uncertainty of the underlying sky map. This uncertainty then represents the systematic uncertainty propagated from the simulated dataset to the calibration factor. Further, we have the $2 \%$ statistical uncertainty propagated from the measured dataset. The results are summarized in Table 1. We studied the propagation of the uncertainty of the voltage calibration constant to the reconstructed electromagnetic energy. We found that this uncertainty is propagating approximately linearly.

We also examine the effects of random uncertainties propagating from the sky maps and the antenna model to the calibration factor. We smeared the sky map by $30 \%$ with Gaussian uncertainties and the antenna model by $20 \%$. We found that this kind of uncertainty is effectively averaged out upon the integration.

By fixing two factors, we investigate the effect of the remaining factor on the calibration constant. Since the obtained value of the effect slightly varies with the chosen factor that we fix, we quote the lowest, highest and the mean of the found values. The results are listed in Table 2. The $\sim 4.5 \%$ effect of the choice of the sky map is consistent with the findings of the LOFAR calibration [9].

${ }^{1}$ LFmap, GSM2008, GSM2016, LFSS and Haslam.

${ }^{2}$ Seven models with different ground conditions, six with shifted components (SSD horizontally shifted by 20, 10, $5 \mathrm{~cm}$, horizontally shifted solar panel by $20 \mathrm{~cm}$, vertically shifted SSD by $10 \mathrm{~cm}$ and horizontally and vertically shifted SSD by $10 \mathrm{~cm}$ ) and four with omitted components (no WCD tank, no SSD, no SSD and solar panel, only antenna). 
Table 1: Calibration constants. The first Table 2: Effect of the different factors (in percents) on the uncertainty is propagated from the simulated calibration constant. *When the LFSS map is fixed, the dataset. The second is the uncertainty propa- methods yield higher inconsistency compared to when the gated from the measured dataset. other maps are used.

\begin{tabular}{cc} 
channel & voltage calibration constant \\
\hline EW & $1.03 \pm 9.6 \% \pm 2.0 \%$ \\
NS & $0.96 \pm 9.7 \% \pm 2.0 \%$
\end{tabular}

\begin{tabular}{lccc} 
factor & $\min$ & $\max$ & mean \\
\hline choice of the sky map & 4.0 & 5.1 & 4.5 \\
choice of calibration method & 1.6 & 5.0 & 3.6 \\
$*$ & $(8.4)$ & $(11.2)$ & $(10.0)$ \\
antenna model & 0.3 & 1.4 & 0.9 \\
antenna - different ground & 0.2 & 1.8 & 1.0 \\
antenna - shifted components & 0.1 & 0.7 & 0.4 \\
antenna - missing components & 0.2 & 1.9 & 0.6
\end{tabular}

The choice of the calibration method results in a $\sim 3.6 \%$ effect. However, this inconsistency is likely due to the low statistics of the data sample. We expect the calibration constants obtained using different calibration methods will be more consistent with higher statistics. When the LFSS map is used, the inconsistency between the results from different calibration methods is much higher than when the other sky maps are used. The antenna model affects the calibration constant weakly, at most $1.9 \%$, which is also consistent with the LOFAR findings (2.5\% [9]).

\section{Measured air showers}

For the cosmic ray studies, we use four RD stations installed in the standard $1500 \mathrm{~m}$ array. To verify that these RD stations are measuring air showers, we reconstruct air showers in the $1500 \mathrm{~m}$ array from 2020/05/14 until 2020/10/21 using the WCDs. We measure 28 showers with a signal in at least one of the four RD stations. The distribution of the azimuth angle of the shower arrival direction is depicted in Fig. 6(a) and radio traces from a shower measured simultaneously by three RD station are shown in Fig. 6(b). Because of the low number of showers, we do not apply quality cuts. We simulate the selected air showers with CORSIKA/CoREAS [19, 20], using the WCD reconstructed air-shower quantities as input. The primary particle was assumed to be a proton. In Fig. 6(c) a comparison of the measured and simulated radio pulse amplitudes is illustrated.

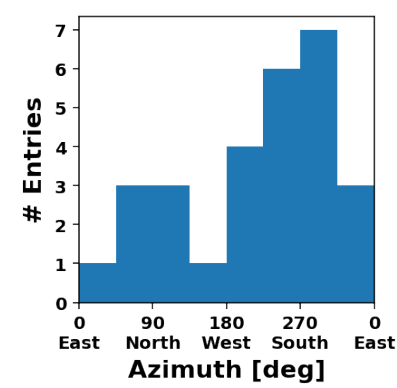

(a)

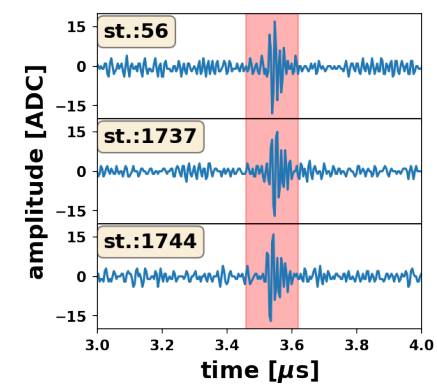

(b)

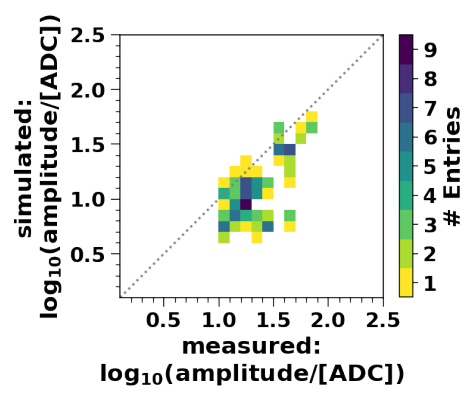

(c)

Figure 6: (a) Azimuth angle distribution of the measured showers. (b) Simultaneously measured radio signals from an air-shower in three RD stations in EW channel. The red band depicts the expected signal position window set by WCD. (c) Correlation between the CoREAS-simulated and the measured radio pulse amplitudes. 
To test for the correlation between these pulses we use Pearson's test. We find a positive correlation $(\mathrm{r}=0.67)$ and rejected the null hypothesis that the compared pulses are uncorrelated at significance level 0.01 (critical value $r_{\text {crit }}=0.22$ ).

\section{Conclusion and outlook}

We presented an end-to-end pilot calibration of the AugerPrime Radio Detector, using the Galactic radio emission and derived the first calibration constants for the RD. We described the necessary steps in the calibration procedure and discussed the effects of various factors on the calibration parameters. The sensitivity of the RD to the Galactic radio emission will allow us to perform an absolute calibration of the full Radio Detector array. Further, we have confirmed that the installed RD engineering array stations are measuring the radio signals from air showers with a satisfying accuracy.

\section{Acknowledgement}

This project is supported by the European Research Council, project number 787622 .

\section{References}

[1] A. Castellina for the Pierre Auger Collaboration, EPJ Web of Conferences 210 (2019) 06002.

[2] J. Rautenberg for the Pierre Auger Collaboration, PoS ICRC2021 (2021) 265.

[3] J.R. Hörandel for the Pierre Auger Collaboration, EPJ Web Conf. 210 (2019) 06005.

[4] J.R. Hörandel for the Pierre Auger Collaboration, EPJ Web Conf. 216 (2019) 01010.

[5] O. Krömer et al., 31st International Cosmic Ray Conference (2009) 1232.

[6] B. Pont for the Pierre Auger Collaboration, PoS ICRC2019 (2019) 395.

[7] F. Schlüter for the Pierre Auger Collaboration, PoS ICRC2021 (2021) 262.

[8] S. He et al., Anal. Methods 6 (2014) 4402.

[9] K. Mulrey et al., Astroparticle Physics 111 (2019) 1.

[10] E. Polisensky, Long WavelengthArray (LWA) Memo Series 111 (2007).

[11] D.C. Price, Astrophysics Source Code Library (2016) [ascl : 1603.013].

[12] J. Dowell et al., Monthly Notices of the Royal Astronomical Society 469 (2017) 4537-4550.

[13] A. de Oliveira-Costa et al., Monthly Notices of the Royal Astronomical Society 388 (2008) 247-260.

[14] H. Zheng et al., Monthly Notices of the Royal Astronomical Society 464 (2016) 3486-3497.

[15] C.G.T. Haslam et al., Astronomy and Astrophysics 47 (1982) 1.

[16] M. Remazeilles et al., Monthly Notices of the Royal Astronomical Society 451 (2015) 4311 [1411.3628].

[17] T. Mozdzen et al., Monthly Notices of the Royal Astronomical Society 483 (2018) 4411.

[18] S. Jansen, Radio for the Masses: Cosmic ray mass composition, Ph.D. thesis, Radboud University Nijmegen, 2016.

[19] D. Heck et al., Tech. Rep. FZKA-6019, Forschungszentrum Karlsruhe (1998).

[20] T. Huege et al., AIP Conf. Proc. 1535 (2013) 128 [1301.2132].

[21] C.R. Harris et al., Nature 585 (2020) 357.

[22] P. Virtanen et al., Nature Methods 17 (2020) 261.

[23] W. McKinney et al., Proceedings of the 9th Python in Science Conference (2010) 56.

[24] M. Newville et al., Zenodo (2014).

[25] J.D. Hunter, Computing in Science \& Engineering 9 (2007) 90.

[26] A. Zonca et al., Journal of Open Source Software 4 (2019) 1298.

This analysis makes use of the following software packages: numpy [21], scipy [22], pandas [23], lmfit [24], matplotlib [25], healpy [26]. 


\section{The Pierre Auger Collaboration}

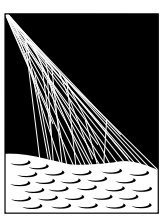

PIERRE

AUSGERVATORY

P. Abreu ${ }^{72}$, M. Aglietta ${ }^{54,52}$, J.M. Albury ${ }^{13}$, I. Allekotte ${ }^{1}$, A. Almela ${ }^{8,12}$, J. Alvarez-Muñiz ${ }^{79}$, R. Alves Batista ${ }^{80}$, G.A. Anastasi ${ }^{63,52}$, L. Anchordoqui ${ }^{87}$, B. Andrada ${ }^{8}$, S. Andringa ${ }^{72}$, C. $\mathrm{Aramo}^{50}$, P.R. Araújo Ferreira ${ }^{42}$, J. C. Arteaga Velázquez ${ }^{67}$, H. Asorey $^{8}$, P. Assis ${ }^{72}$, G. Avila ${ }^{11}$, A.M. Badescu ${ }^{75}$, A. Bakalova ${ }^{32}$, A. Balaceanu ${ }^{73}$, F. Barbato ${ }^{45,46}$, R.J. Barreira Luz $^{72}$, K.H. Becker ${ }^{38}$, J.A. Bellido ${ }^{13,69}$, C. Berat ${ }^{36}$, M.E. Bertaina ${ }^{63,52}$, X. Bertou ${ }^{1}$, P.L. Biermann ${ }^{b}$, V. Binet ${ }^{6}$, K. Bismark ${ }^{39,8}$, T. Bister ${ }^{42}$, J. Biteau ${ }^{37}$, J. Blazek ${ }^{32}$, C. Bleve ${ }^{36}$, M. Boháčová ${ }^{32}$, D. Boncioli ${ }^{57,46}$, C. Bonifazi ${ }^{9,26}$, L. Bonneau Arbeletche ${ }^{21}$, N. Borodai ${ }^{70}$, A.M. Botti ${ }^{8}$, J. Brack ${ }^{d}$, T. Bretz ${ }^{42}$, P.G. Brichetto Orchera ${ }^{8}$, F.L. Briechle ${ }^{42}$, P. Buchholz ${ }^{44}$, A. Bueno ${ }^{78}$, S. Buitink ${ }^{15}$, M. Buscemi ${ }^{47}$, M. Büsken ${ }^{39,8}$, K.S. Caballero-Mora ${ }^{66}$, L. Caccianiga ${ }^{59,49}$, F. Canfora ${ }^{80,81}$, I. Caracas ${ }^{38}$, J.M. Carceller ${ }^{78}$, R. Caruso ${ }^{58,47}$, A. Castellina ${ }^{54,52}$, F. Catalanii ${ }^{19}$, G. Cataldi ${ }^{48}$, L. Cazon $^{72}$, M. Cerda ${ }^{10}$, J.A. Chinellato ${ }^{22}$, J. Chudoba ${ }^{32}$, L. Chytka ${ }^{33}$, R.W. Clay ${ }^{13}$, A.C. Cobos Cerutti ${ }^{7}$, R. Colalillo ${ }^{60,50}$, A. Coleman ${ }^{93}$, M.R. Coluccia ${ }^{48}$, R. Conceição ${ }^{72}$, A. Condorellii ${ }^{45,46}$, G. Consolati ${ }^{49,55}$, F. Contreras ${ }^{11}$, F. Convenga ${ }^{56,48}$, D. Correia dos Santos $^{28}$, C.E. Covault ${ }^{85}$, S. Dasso ${ }^{5,3}$, K. Daumiller ${ }^{41}$, B.R. Dawson ${ }^{13}$, J.A. Day ${ }^{13}$, R.M. de Almeida ${ }^{28}$, J. de Jesús ${ }^{8,41}$, S.J. de Jong ${ }^{80,81}$, G. De Mauro ${ }^{80,81}$, J.R.T. de Mello Neto ${ }^{26,27}$, I. De Mitri ${ }^{45,46}$, J. de Oliveira ${ }^{18}$, D. de Oliveira Franco ${ }^{22}$, F. de Palma ${ }^{56,48}$, V. de Souza $^{20}$, E. De Vito ${ }^{56,48}$, M. del Río ${ }^{11}$, O. Deligny ${ }^{34}$, L. Deval ${ }^{41,8}$, A. di Matteo $^{52}$, C. Dobrigkeit ${ }^{22}$, J.C. D'Olivo ${ }^{68}$, L.M. Domingues Mendes ${ }^{72}$, R.C. dos Anjos ${ }^{25}$, D. dos Santos ${ }^{28}$, M.T. Dova ${ }^{4}$, J. Ebr ${ }^{32}$, R. Engel ${ }^{39,41}$, I. Epicoco ${ }^{56,48}$, M. Erdmann $^{42}$, C.O. Escobar ${ }^{a}$, A. Etchegoyen ${ }^{8,12}$, H. Falcke ${ }^{80,82,81}$, J. Farmer ${ }^{92}$, G. Farrar ${ }^{90}$, A.C. Fauth ${ }^{22}$, N. Fazzini ${ }^{a}$, F. Feldbusch ${ }^{40}$, F. Fenu ${ }^{54,52}$, B. Fick ${ }^{89}$, J.M. Figueira ${ }^{8}$, A. Filipčíc $\check{c}^{77,76}$, T. Fitoussi ${ }^{41}$, T. Fodran ${ }^{80}$, M.M. Freire ${ }^{6}$, T. Fujii9 ${ }^{22, e}$, A. Fuster ${ }^{8,12}$, C. Galea ${ }^{80}$, C. Galellii ${ }^{59,49}$, B. García ${ }^{7}$, A.L. Garcia Vegas ${ }^{42}$, H. Gemmeke ${ }^{40}$, F. Gesualdi ${ }^{8,41}$, A. Gherghel-Lascu ${ }^{73}$, P.L. Ghia ${ }^{34}$, U. Giaccari ${ }^{80}$, M. Giammarchi ${ }^{49}$, J. Glombitza ${ }^{42}$, F. Gobbi ${ }^{10}$, F. Gollan ${ }^{8}$, G. Golup ${ }^{1}$, M. Gómez Berisso ${ }^{1}$, P.F. Gómez Vitale ${ }^{11}$, J.P. Gongora ${ }^{11}$, J.M. González ${ }^{1}$, N. González ${ }^{14}$, I. Goos ${ }^{1,41}$, D. Góra ${ }^{70}$, A. Gorgi ${ }^{54,52}$, M. Gottowik ${ }^{38}$, T.D. Grubb ${ }^{13}$, F. Guarino ${ }^{60,50}$, G.P. Guedes ${ }^{23}$, E. Guido ${ }^{52,63}$, S. Hahn ${ }^{41,8}$, P. Hamal ${ }^{32}$, M.R. Hampel ${ }^{8}$, P. Hansen ${ }^{4}$, D. Harari ${ }^{1}$, V.M. Harvey ${ }^{13}$, A. Haungs ${ }^{41}$, T. Hebbeker ${ }^{42}$, D. Heck ${ }^{41}$, G.C. Hill ${ }^{13}$, C. Hojvat ${ }^{a}$, J.R. Hörandel ${ }^{80,81}$, P. Horvath ${ }^{33}$, M. Hrabovský $\dot{y}^{33}$, T. Huege ${ }^{41,15}$, A. Insolia ${ }^{58,47}$, P.G. Isar ${ }^{74}$, P. Janecek ${ }^{32}$, J.A. Johnsen ${ }^{86}$, J. Jurysek ${ }^{32}$, A. Kääpä ${ }^{38}$, K.H. Kampert ${ }^{38}$, N. Karastathis ${ }^{41}$, B. Keilhauer ${ }^{41}$, J. Kemp ${ }^{42}$, A. Khakurdikar ${ }^{80}$, V.V. Kizakke Covilakam $^{8,41}$, H.O. Klages ${ }^{41}$, M. Kleifges ${ }^{40}$, J. Kleinfeller ${ }^{10}$, M. Köpke ${ }^{39}$, N. Kunka ${ }^{40}$, B.L. Lago ${ }^{17}$, R.G. Lang ${ }^{20}$, N. Langner ${ }^{42}$, M.A. Leigui de Oliveira ${ }^{24}$, V. Lenok ${ }^{41}$, A. Letessier-Selvon ${ }^{35}$, I. LhenryYvon $^{34}$, D. Lo Presti ${ }^{58,47}$, L. Lopes ${ }^{72}$, R. López ${ }^{64}$, L. Lu ${ }^{94}$, Q. Luce ${ }^{39}$, J.P. Lundquist ${ }^{76}$, A. Machado

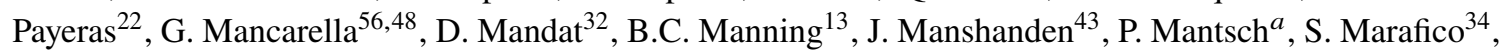
A.G. Mariazzi ${ }^{4}$, I.C. Mariş ${ }^{14}$, G. Marsella ${ }^{61,47}$, D. Martello ${ }^{56,48}$, S. Martinelliili,8, O. Martínez Bravo ${ }^{64}$, M. Mastrodicasa ${ }^{57,46}$, H.J. Mathes ${ }^{41}$, J. Matthews ${ }^{88}$, G. Matthiae ${ }^{62,51}$, E. Mayotte ${ }^{38}$, P.O. Mazur ${ }^{a}$, G. MedinaTanco $^{68}$, D. Melo $^{8}$, A. Menshikov ${ }^{40}$, K.-D. Merenda ${ }^{86}$, S. Michal ${ }^{33}$, M.I. Micheletti ${ }^{6}$, L. Miramonti ${ }^{59,49}$, S. Mollerach ${ }^{1}$, F. Montanet ${ }^{36}$, C. Morello ${ }^{54,52}$, M. Mostafá ${ }^{91}$, A.L. Müller ${ }^{8}$, M.A. Muller ${ }^{22}$, K. Mulrey ${ }^{15}$, R. Mussa ${ }^{52}$, M. Muzio ${ }^{90}$, W.M. Namasaka ${ }^{38}$, A. Nasr-Esfahani ${ }^{38}$, L. Nellen $^{68}$, M. Niculescu-Oglinzanu ${ }^{73}$, M. Niechciol ${ }^{44}$, D. Nitz ${ }^{89}$, D. Nosek ${ }^{31}$, V. Novotny ${ }^{31}$, L. Nožka ${ }^{33}$, A Nucita ${ }^{56,48}$, L.A. Núñez ${ }^{30}$, M. Palatka ${ }^{32}$, J. Pallotta ${ }^{2}$, P. Papenbreer ${ }^{38}$, G. Parente ${ }^{79}$, A. Parra ${ }^{64}$, J. Pawlowsky ${ }^{38}$, M. Pech ${ }^{32}$, F. Pedreira ${ }^{79}$, J. Pękala ${ }^{70}$, R. Pelayo ${ }^{65}$, J. Peña-Rodriguez ${ }^{30}$, E.E. Pereira Martins ${ }^{39,8}$, J. Perez Armand ${ }^{21}$, C. Pérez

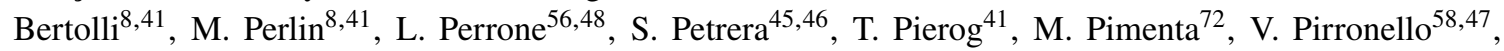
M. Platino ${ }^{8}$, B. Pont $^{80}$, M. Pothast ${ }^{81,80}$, P. Privitera ${ }^{92}$, M. Prouza $^{32}$, A. Puyleart ${ }^{89}$, S. Querchfeld ${ }^{38}$, J. Rautenberg ${ }^{38}$, D. Ravignani ${ }^{8}$, M. Reininghaus ${ }^{41,8}$, J. Ridky ${ }^{32}$, F. Riehn ${ }^{72}$, M. Risse ${ }^{44}$, V. Rizi ${ }^{57,46}$, W. Rodrigues de Carvalho ${ }^{21}$, J. Rodriguez Rojo ${ }^{11}$, M.J. Roncoroni ${ }^{8}$, S. Rossoni ${ }^{43}$, M. Roth ${ }^{41}$, E. Roulet ${ }^{1}$, A.C. Rovero ${ }^{5}$, P. Ruehl ${ }^{44}$, A. Saftoiu ${ }^{73}$, F. Salamida ${ }^{57,46}$, H. Salazar ${ }^{64}$, G. Salina ${ }^{51}$, J.D. Sanabria Gomez ${ }^{30}$, F. Sánchez ${ }^{8}$, E.M. Santos ${ }^{21}$, E. Santos ${ }^{32}$, F. Sarazin ${ }^{86}$, R. Sarmento ${ }^{72}$, C. Sarmiento-Cano ${ }^{8}$, R. Sato ${ }^{11}$, P. Savina ${ }^{56,48,34,94}$, C.M. Schäfer ${ }^{41}$, V. Scherini ${ }^{56,48}$, H. Schieler ${ }^{41}$, M. Schimassek ${ }^{39,8}$, M. Schimp ${ }^{38}$, 
F. Schlüter ${ }^{41,8}$, D. Schmidt ${ }^{39}$, O. Scholten ${ }^{84,15}$, P. Schovánek ${ }^{32}$, F.G. Schröder ${ }^{93,41}$, S. Schröder ${ }^{38}$, J. Schulte ${ }^{42}$, S.J. Sciutto ${ }^{4}$, M. Scornavacche ${ }^{8,41}$, A. Segreto ${ }^{53,47}$, S. Sehgal ${ }^{38}$, R.C. Shellard ${ }^{16}$, G. Sigl ${ }^{43}$, G. Silli ${ }^{8,41}$, O. Sima ${ }^{73, f}$, R. Šmída ${ }^{92}$, P. Sommers ${ }^{91}$, J.F. Soriano ${ }^{87}$, J. Souchard ${ }^{36}$, R. Squartini ${ }^{10}$, M. Stadelmaier ${ }^{41,8}$, D. Stanca ${ }^{73}$, S. Staničc ${ }^{76}$, J. Stasielak ${ }^{70}$, P. Stassi ${ }^{36}$, A. Streich ${ }^{39,8}$, M. Suárez-Durán ${ }^{14}$, T. Sudholz ${ }^{13}$, T. Suomijärvi ${ }^{37}$, A.D. Supanitsky ${ }^{8}$, Z. Szadkowski ${ }^{71}$, A. Tapia ${ }^{29}$, C. Taricco ${ }^{63,52}$, C. Timmermans ${ }^{81,80}$, O. Tkachenko ${ }^{41}$, P. Tobiska ${ }^{32}$, C.J. Todero Peixoto ${ }^{19}$, B. Tomé ${ }^{72}$, Z. Torrès ${ }^{36}$, A. Travaini ${ }^{10}$, P. Travnicek ${ }^{32}$, C. Trimarelli ${ }^{57,46}$, M. Tueros ${ }^{4}$, R. Ulrich ${ }^{41}$, M. Unger ${ }^{41}$, L. Vaclavek ${ }^{33}$, M. Vacula ${ }^{33}$, J.F. Valdés Galicia ${ }^{68}$, L. Valore ${ }^{60,50}$, E. Varela ${ }^{64}$, A. Vásquez-Ramírez ${ }^{30}$, D. Veberič ${ }^{41}$, C. Ventura ${ }^{27}$, I.D. Vergara Quispe ${ }^{4}$, V. Verzi ${ }^{51}$, J. Vicha ${ }^{32}$, J. Vink ${ }^{83}$, S. Vorobiov ${ }^{76}$, H. Wahlberg ${ }^{4}$, C. Watanabe ${ }^{26}$, A.A. Watson ${ }^{c}$, M. Weber ${ }^{40}$, A. Weindl ${ }^{41}$, L. Wiencke ${ }^{86}$, H. Wilczyński ${ }^{70}$, M. Wirtz ${ }^{42}$, D. Wittkowski ${ }^{38}$, B. Wundheiler ${ }^{8}$, A. Yushkov $^{32}$, O. Zapparrata ${ }^{14}$, E. Zas ${ }^{79}$, D. Zavrtanik ${ }^{76,77}$, M. Zavrtanik ${ }^{77,76}$, L. Zehrer ${ }^{76}$

${ }^{1}$ Centro Atómico Bariloche and Instituto Balseiro (CNEA-UNCuyo-CONICET), San Carlos de Bariloche, Argentina

${ }^{2}$ Centro de Investigaciones en Láseres y Aplicaciones, CITEDEF and CONICET, Villa Martelli, Argentina

${ }^{3}$ Departamento de Física and Departamento de Ciencias de la Atmósfera y los Océanos, FCEyN, Universidad de Buenos Aires and CONICET, Buenos Aires, Argentina

${ }^{4}$ IFLP, Universidad Nacional de La Plata and CONICET, La Plata, Argentina

${ }^{5}$ Instituto de Astronomía y Física del Espacio (IAFE, CONICET-UBA), Buenos Aires, Argentina

${ }^{6}$ Instituto de Física de Rosario (IFIR) - CONICET/U.N.R. and Facultad de Ciencias Bioquímicas y Farmacéuticas U.N.R., Rosario, Argentina

${ }^{7}$ Instituto de Tecnologías en Detección y Astropartículas (CNEA, CONICET, UNSAM), and Universidad Tecnológica Nacional - Facultad Regional Mendoza (CONICET/CNEA), Mendoza, Argentina

${ }^{8}$ Instituto de Tecnologías en Detección y Astropartículas (CNEA, CONICET, UNSAM), Buenos Aires, Argentina

${ }^{9}$ International Center of Advanced Studies and Instituto de Ciencias Físicas, ECyT-UNSAM and CONICET, Campus Miguelete - San Martín, Buenos Aires, Argentina

${ }^{10}$ Observatorio Pierre Auger, Malargüe, Argentina

11 Observatorio Pierre Auger and Comisión Nacional de Energía Atómica, Malargüe, Argentina

12 Universidad Tecnológica Nacional - Facultad Regional Buenos Aires, Buenos Aires, Argentina

${ }^{13}$ University of Adelaide, Adelaide, S.A., Australia

${ }^{14}$ Université Libre de Bruxelles (ULB), Brussels, Belgium

15 Vrije Universiteit Brussels, Brussels, Belgium

${ }^{16}$ Centro Brasileiro de Pesquisas Fisicas, Rio de Janeiro, RJ, Brazil

${ }^{17}$ Centro Federal de Educação Tecnológica Celso Suckow da Fonseca, Nova Friburgo, Brazil

${ }^{18}$ Instituto Federal de Educação, Ciência e Tecnologia do Rio de Janeiro (IFRJ), Brazil

${ }^{19}$ Universidade de São Paulo, Escola de Engenharia de Lorena, Lorena, SP, Brazil

${ }^{20}$ Universidade de São Paulo, Instituto de Física de São Carlos, São Carlos, SP, Brazil

${ }^{21}$ Universidade de São Paulo, Instituto de Física, São Paulo, SP, Brazil

22 Universidade Estadual de Campinas, IFGW, Campinas, SP, Brazil

${ }^{23}$ Universidade Estadual de Feira de Santana, Feira de Santana, Brazil

${ }^{24}$ Universidade Federal do ABC, Santo André, SP, Brazil

25 Universidade Federal do Paraná, Setor Palotina, Palotina, Brazil

${ }^{26}$ Universidade Federal do Rio de Janeiro, Instituto de Física, Rio de Janeiro, RJ, Brazil

${ }^{27}$ Universidade Federal do Rio de Janeiro (UFRJ), Observatório do Valongo, Rio de Janeiro, RJ, Brazil

28 Universidade Federal Fluminense, EEIMVR, Volta Redonda, RJ, Brazil

${ }^{29}$ Universidad de Medellín, Medellín, Colombia

${ }^{30}$ Universidad Industrial de Santander, Bucaramanga, Colombia

${ }^{31}$ Charles University, Faculty of Mathematics and Physics, Institute of Particle and Nuclear Physics, Prague, Czech Republic

32 Institute of Physics of the Czech Academy of Sciences, Prague, Czech Republic

${ }^{33}$ Palacky University, RCPTM, Olomouc, Czech Republic 
${ }^{34}$ CNRS/IN2P3, IJCLab, Université Paris-Saclay, Orsay, France

${ }^{35}$ Laboratoire de Physique Nucléaire et de Hautes Energies (LPNHE), Sorbonne Université, Université de Paris, CNRSIN2P3, Paris, France

${ }^{36}$ Univ. Grenoble Alpes, CNRS, Grenoble Institute of Engineering Univ. Grenoble Alpes, LPSC-IN2P3, 38000 Grenoble, France

${ }^{37}$ Université Paris-Saclay, CNRS/IN2P3, IJCLab, Orsay, France

38 Bergische Universität Wuppertal, Department of Physics, Wuppertal, Germany

${ }^{39}$ Karlsruhe Institute of Technology (KIT), Institute for Experimental Particle Physics, Karlsruhe, Germany

${ }^{40}$ Karlsruhe Institute of Technology (KIT), Institut für Prozessdatenverarbeitung und Elektronik, Karlsruhe, Germany

${ }^{41}$ Karlsruhe Institute of Technology (KIT), Institute for Astroparticle Physics, Karlsruhe, Germany

${ }^{42}$ RWTH Aachen University, III. Physikalisches Institut A, Aachen, Germany

${ }^{43}$ Universität Hamburg, II. Institut für Theoretische Physik, Hamburg, Germany

${ }^{44}$ Universität Siegen, Department Physik - Experimentelle Teilchenphysik, Siegen, Germany

${ }^{45}$ Gran Sasso Science Institute, L’Aquila, Italy

${ }^{46}$ INFN Laboratori Nazionali del Gran Sasso, Assergi (L’Aquila), Italy

${ }^{47}$ INFN, Sezione di Catania, Catania, Italy

${ }^{48}$ INFN, Sezione di Lecce, Lecce, Italy

${ }^{49}$ INFN, Sezione di Milano, Milano, Italy

50 INFN, Sezione di Napoli, Napoli, Italy

51 INFN, Sezione di Roma "Tor Vergata”, Roma, Italy

52 INFN, Sezione di Torino, Torino, Italy

53 Istituto di Astrofisica Spaziale e Fisica Cosmica di Palermo (INAF), Palermo, Italy

54 Osservatorio Astrofisico di Torino (INAF), Torino, Italy

55 Politecnico di Milano, Dipartimento di Scienze e Tecnologie Aerospaziali , Milano, Italy

56 Università del Salento, Dipartimento di Matematica e Fisica “E. De Giorgi”, Lecce, Italy

${ }^{57}$ Università dell'Aquila, Dipartimento di Scienze Fisiche e Chimiche, L’Aquila, Italy

${ }^{58}$ Università di Catania, Dipartimento di Fisica e Astronomia, Catania, Italy

${ }^{59}$ Università di Milano, Dipartimento di Fisica, Milano, Italy

${ }^{60}$ Università di Napoli "Federico II", Dipartimento di Fisica "Ettore Pancini”, Napoli, Italy

${ }^{61}$ Università di Palermo, Dipartimento di Fisica e Chimica "E. Segrè”, Palermo, Italy

62 Università di Roma "Tor Vergata", Dipartimento di Fisica, Roma, Italy

${ }^{63}$ Università Torino, Dipartimento di Fisica, Torino, Italy

64 Benemérita Universidad Autónoma de Puebla, Puebla, México

${ }^{65}$ Unidad Profesional Interdisciplinaria en Ingeniería y Tecnologías Avanzadas del Instituto Politécnico Nacional (UPIITA-IPN), México, D.F., México

66 Universidad Autónoma de Chiapas, Tuxtla Gutiérrez, Chiapas, México

${ }^{67}$ Universidad Michoacana de San Nicolás de Hidalgo, Morelia, Michoacán, México

68 Universidad Nacional Autónoma de México, México, D.F., México

${ }^{69}$ Universidad Nacional de San Agustin de Arequipa, Facultad de Ciencias Naturales y Formales, Arequipa, Peru

${ }^{70}$ Institute of Nuclear Physics PAN, Krakow, Poland

${ }^{71}$ University of Łódź, Faculty of High-Energy Astrophysics,Lódź, Poland

${ }^{72}$ Laboratório de Instrumentação e Física Experimental de Partículas - LIP and Instituto Superior Técnico - IST, Universidade de Lisboa - UL, Lisboa, Portugal

73 "Horia Hulubei” National Institute for Physics and Nuclear Engineering, Bucharest-Magurele, Romania

${ }^{74}$ Institute of Space Science, Bucharest-Magurele, Romania

75 University Politehnica of Bucharest, Bucharest, Romania

${ }^{76}$ Center for Astrophysics and Cosmology (CAC), University of Nova Gorica, Nova Gorica, Slovenia

${ }^{77}$ Experimental Particle Physics Department, J. Stefan Institute, Ljubljana, Slovenia

${ }^{78}$ Universidad de Granada and C.A.F.P.E., Granada, Spain

${ }^{79}$ Instituto Galego de Física de Altas Enerxías (IGFAE), Universidade de Santiago de Compostela, Santiago de Compostela, Spain

${ }^{80}$ IMAPP, Radboud University Nijmegen, Nijmegen, The Netherlands 
${ }^{81}$ Nationaal Instituut voor Kernfysica en Hoge Energie Fysica (NIKHEF), Science Park, Amsterdam, The Netherlands

${ }^{82}$ Stichting Astronomisch Onderzoek in Nederland (ASTRON), Dwingeloo, The Netherlands

${ }^{83}$ Universiteit van Amsterdam, Faculty of Science, Amsterdam, The Netherlands

${ }^{84}$ University of Groningen, Kapteyn Astronomical Institute, Groningen, The Netherlands

${ }^{85}$ Case Western Reserve University, Cleveland, OH, USA

${ }^{86}$ Colorado School of Mines, Golden, CO, USA

${ }^{87}$ Department of Physics and Astronomy, Lehman College, City University of New York, Bronx, NY, USA

${ }^{88}$ Louisiana State University, Baton Rouge, LA, USA

${ }^{89}$ Michigan Technological University, Houghton, MI, USA

${ }^{90}$ New York University, New York, NY, USA

${ }^{91}$ Pennsylvania State University, University Park, PA, USA

92 University of Chicago, Enrico Fermi Institute, Chicago, IL, USA

93 University of Delaware, Department of Physics and Astronomy, Bartol Research Institute, Newark, DE, USA

94 University of Wisconsin-Madison, Department of Physics and WIPAC, Madison, WI, USA

${ }^{a}$ Fermi National Accelerator Laboratory, Fermilab, Batavia, IL, USA

${ }^{b}$ Max-Planck-Institut für Radioastronomie, Bonn, Germany

${ }^{c}$ School of Physics and Astronomy, University of Leeds, Leeds, United Kingdom

${ }^{d}$ Colorado State University, Fort Collins, CO, USA

$e^{e}$ now at Hakubi Center for Advanced Research and Graduate School of Science, Kyoto University, Kyoto, Japan

$f$ also at University of Bucharest, Physics Department, Bucharest, Romania 